\title{
Higher-dimensional generalizations of Berry curvature
}

\author{
Anton Kapustin* and Lev Spodyneiko ${ }^{*}$ \\ California Institute of Technology, Pasadena, California 91125, USA
}

(Received 20 February 2020; accepted 14 May 2020; published 11 June 2020)

\begin{abstract}
A family of finite-dimensional quantum systems with a nondegenerate ground state gives rise to a closed two-form on the parameter space, the curvature of the Berry connection. Its integral over a surface detects the presence of degeneracy points inside the volume enclosed by the surface. We seek generalizations of the Berry curvature to gapped many-body systems in $D$ spatial dimensions which can detect gapless or degenerate points in the phase diagram of a system. Field theory predicts that in spatial dimension $D$ the analog of the Berry curvature is a closed $(D+2)$-form on the parameter space (the Wess-Zumino-Witten form). We construct such closed forms for arbitrary families of gapped interacting lattice systems in all dimensions. We show that whenever the integral of the Wess-Zumino-Witten form over a $(D+2)$-dimensional surface in the parameter space is nonzero, there must be gapless edge modes for at least one value of the parameters. These edge modes arise even when the bulk system is in a trivial phase for all values of the parameters and are protected by the nontrivial topology of the phase diagram.
\end{abstract}

DOI: 10.1103/PhysRevB.101.235130

\section{INTRODUCTION}

Consider a quantum-mechanical system with a Hamiltonian depending on parameters, a unique ground state for all values of the parameters, and an energy gap to the lowest excited state. To these data, one can associate a differential two-form $\Omega$ on the parameter space: The curvature of the Berry connection [1]. This two-form is closed and quantized: Its integrals over closed surfaces are integral multiples of $2 \pi$. If the integral of $\Omega$ over a surface $\Sigma$ is nonzero, $\Sigma$ must enclose points where the ground state is degenerate. Indeed, if the system were nondegenerate everywhere inside $\Sigma$, then by Stokes' theorem the integral of $\Omega$ over $\Sigma$ would vanish. Thus, the Berry curvature can detect degeneracy points. Moreover, degeneracy points detected by the Berry curvature are stable against deformations of the Hamiltonian. Indeed, since the integral of the Berry curvature is quantized, it can only change discontinuously and thus any small deformation will not affect the value of the integral.

When we consider many-body Hamiltonians, the most direct analogs of Hamiltonians with a unique nondegenerate ground state are gapped systems in a trivial phase or, more generally, systems in a short-range entangled (SRE) phase. The analogs of degeneracy points are points where phase transitions occur. In view of the above discussion, it is natural to ask whether the presence of a phase transition (either continuous or discontinuous) can be detected by studying SRE systems in the neighborhood of the suspected phase transition. By analogy with the above discussion, one would hope to construct closed forms on the parameter space of SRE systems whose integrals over surfaces would serve as signatures of phase transitions.

\footnotetext{
*kapustin@theory.caltech.edu

$\dagger$ lionspo@caltech.edu
}

A few years ago, Kitaev [2] proposed that, for a family of SRE systems in spatial dimensions $D$, one can define a closed $(D+2)$-form on the parameter space. This form is a higher-dimensional generalization of the Berry curvature and can be used to detect phase transition loci in the parameter space. One difficulty in making this proposal concrete is that currently there is no useful definition of SRE systems beyond the "negative" statement that these are systems which exhibit neither spontaneous symmetry breaking nor topological order.

Unlike the notion of a SRE system, the notion of a gapped system is straightforward to define. In this paper, we define and study higher-dimensional generalizations of the Berry curvature for gapped lattice systems on $\mathbb{R}^{D}$. For any gapped lattice Hamiltonian, depending on parameters, we define a closed $(D+2)$-form $\Omega^{(D+2)}$ on the parameter space. ${ }^{1}$ If an integral of this form over a $(D+2)$-dimensional surface in the parameter space is nonzero, then this surface cannot be contracted to a point within the space of gapped systems. Thus, such integrals are able to detect the presence of phase transition loci completely surrounded by a gapped phase. The analogy with the Berry curvature is most complete in the case when this phase is SRE, as in Kitaev's proposal. In that case, one can argue that the integral of $\Omega^{(D+2)}$ over any $(D+2)$ dimensional sphere is quantized (is an integral multiple of $2 \pi)$.

In spatial dimension $D>0$, it is natural to study boundaries or interfaces. It is well-known by now that an interface between two gapped systems in different phases may host gapless modes which are robust with respect to all

\footnotetext{
${ }^{1}$ For families of Euclidean lattice systems in $D+1$ dimensions with exponentially decaying correlations, Kitaev outlined a construction of a closed $(D+2)$-form on the parameter space [2]. Our results can be viewed as a Hamiltonian version of this construction.
} 
deformations (or all deformations which do not break the symmetry, if one is dealing with symmetry-protected topological phases).

If one considers an interface between two gapped systems in the same phase, then it is generically gapped. Even if there are gapless modes on a particular interface, a small deformation of the Hamiltonian can make them gapped. But the situation becomes more interesting if one considers families of trivially gapped systems and interfaces between them. Following the ideology of catastrophe theory [3], one might expect that sometimes perturbing the family cannot eliminate a gapless interface; it merely moves it to a different location in the parameter space. We show that the form $\Omega^{(D+2)}$ can serve as a diagnostic for such families. Namely, suppose we are given a family of gapped $D$-dimensional Hamiltonians $H\left(\lambda_{1}, \ldots, \lambda_{D+2}\right)$ continuously depending on $D+2$ parameters, such that the parameter space is a closed oriented $(D+$ 2)-dimensional manifold. Fix a Hamiltonian $H_{0}$ in the same phase as all the Hamiltonians in the family [for example, one can just let $H_{0}$ be $H\left(\lambda_{1}, \ldots, \lambda_{D+2}\right)$ for some specific values of the parameters]. Now suppose we are given a family of interfaces between all the systems in the family and the system with the Hamiltonian $H_{0}$. We show that if all the interfaces have a gapped nondegenerate ground state, then the integral of $\Omega^{(D+2)}$ over the parameter space must vanish. This is a form of bulk-boundary correspondence for families.

In view of the above, it is interesting to give examples of families of Hamiltonians where the forms $\Omega^{(D+2)}$ and their integrals are nonzero. We will call such families topologically nontrivial. In general, computing the forms $\Omega^{(D+2)}$ is a difficult task (this is also true for the Berry curvature). But in the case of translationally invariant tight-binding free-fermion Hamiltonians in 1D, we show that the cohomology class of $\Omega^{(D+2)}$ is determined by the curvature of the Berry-Bloch connection. We conjecture that this is true in any dimension. Free-fermion systems thus can provide examples of families which exhibit gapless edge modes in families, despite being in a trivial phase for all values of the parameters.

Recently Cordova et al. studied field theories with "anomalies in the space of couplings" [4,5]. Via the bulk-boundary correspondence, this subject is closely related to topologically nontrivial families of gapped field theories in one dimension higher. It is natural to conjecture that there is a $1: 1$ correspondence between topological invariants of families of gapped field theories in $(D+1)$ space-time dimensions and topological invariants of families of gapped lattice models in $D$ spatial dimensions, some of which we study here.

The content of the paper as follows. In Sec. II, we interpret higher Berry curvature forms in the language of quantum field theory, specifically as Wess-Zumino-Witten terms in the effective action for the parameters. This serves as a motivation for subsequent discussion. In Sec. III, we show how to associate a closed three-form to a family of gapped 1D lattice systems. We show that this three-form can serve as diagnostic for gapless interfaces which are robust for topological reasons. Such gapless modes are rather surprising in bosonic 1D systems without any symmetries. In Sec. IV, we extend our construction to families of gapped lattice systems in arbitrary spatial dimension. This requires some mathematical machinery which we review. We discuss our results in
Sec. V. In Appendix A, we argue that if all systems in the family are SRE, the integral of $\Omega^{(D+2)}$ over any sphere in the parameter space is an integral multiple of $2 \pi$. In Appendix B, we compute the three-form $\Omega^{(3)}$ for families of tight-binding free-fermion 1D systems of class A and relate it to the BerryBloch connection. This allows us to give examples of families of systems where our higher Berry curvature is topologically nontrivial (lies in nonzero cohomology classes).

\section{EFFECTIVE ACTION CONSIDERATIONS}

The purpose of this section is to motivate the constructions in subsequent sections. It is not essential for understanding the rest of the paper. Readers not familiar with topological aspects of Quantum Field Theory (QFT) are advised to skip it on first reading.

If a gapped system in $D$ spatial dimensions is described by a trivial topological field theory at long distances, then its low-energy effective action is a well-defined function of background fields, such as the metric and the gauge fields which couple to global symmetries. If one deals with a family of such systems parameterized by a manifold $\mathrm{M}$, one can let the parameters vary slowly from point to point, and the effective action is still a well-defined function of the background fields. The variation of the parameters can be described by a map $\phi: X \rightarrow \mathrm{M}$, where $X$ is the space-time. The effective action depends on $\phi$ as well as other background fields.

Loosely speaking, topological terms in the action are those terms which survive when one rescales the metric $g_{\mu v} \mapsto$ $e^{\sigma} g_{\mu \nu}$ and takes the limit $\sigma \rightarrow+\infty$. The simplest such terms are those which depend only on $\phi$ and not on other background fields. For example, for $D=0$ (ordinary quantum mechanics), such a topological term schematically has the form

$$
S_{\text {top }}(X, \phi)=\int_{X} \omega_{j}^{(1)} \partial_{t} \phi^{j} d t=\int_{X} \phi^{*}\left(\omega^{(1)}\right),
$$

where $X$ is a $1 \mathrm{D}$ manifold $\left(S^{1}\right.$ or $\left.\mathbb{R}\right)$ and $\omega^{(1)}$ is the one-form on $\mathrm{M}$ representing the Berry connection. In a chart of $\mathrm{M}$ with coordinates $\lambda^{j}$, the one-form is given by $\omega^{(1)}=\sum_{j} \omega_{j}^{(1)} d \lambda^{j}$ and the Berry connection $\omega_{j}^{(1)}=i\left\langle 0_{\lambda}\left|\frac{\partial}{\partial \lambda^{j}}\right| 0_{\lambda}\right\rangle$, where $\left|0_{\lambda}\right\rangle$ is the ground state. The formula Eq. (1) is only schematic because, in general, the Berry connection on the parameter space can be represented by a one-form $\omega^{(1)}$ only locally on $M$. If the Chern class of the Berry curvature $\Omega^{(2)}$ is nontrivial, then one cannot write $\Omega^{(2)}=d \omega^{(1)}$ for a globally defined one-form $\omega^{(1)}$. Rather, one needs to cover the parameter space with charts, in each of which the connection is represented by a one-form. On the overlaps of the charts, these one-forms are related by gauge transformations. To define $S_{\text {top }}(X, \phi)$ properly, one needs to know both the locally defined oneforms and the gauge transformations connecting them.

If the space-time $X$ is circle $S^{1}$, the geometric phase $\exp \left(i S_{\text {top }}(X, \phi)\right)$ represents the phase factor acquired by the ground state under adiabatic transformation around a loop $\phi: S^{1} \rightarrow \mathrm{M}$ in the parameter space. It can be defined unambiguously, while the phase $S_{\text {top }}(X, \phi)$ is defined only up to an integer multiple of $2 \pi$. If the loop in the parameter space anchors a disk $D$, i.e., the map $\phi: X \rightarrow \mathrm{M}$ extends to a continuous map $\tilde{\phi}: D \rightarrow \mathrm{M}$, then one can rewrite the 
geometric phase as integral of the Berry curvature over the surface bounded by the loop:

$$
S_{\text {top }}=\int_{D} \tilde{\phi}^{*}\left(\Omega^{(2)}\right) .
$$

This expression depends on the choice of $\tilde{\phi}$. But $\exp \left(i S_{\text {top }}(X, \phi)\right)$ is unambiguously defined since periods of $\Omega^{(2)}$ are "quantized": The integral of $\Omega^{(2)}$ over any two-cycle on $M$ is $2 \pi$ times an integer.

The geometric phase Eq. (2) depends on the dynamical detail of the system and can always be made trivial on any contractable submanifold of $\mathrm{M}$ by a suitable deformation of the Hamiltonian. A nonzero value of the integral of the Berry curvature over the closed surface indicates the presence of a gapless point inside of it. The latter serves as an obstruction for the contraction of this surface. More generally, any globally defined Berry connection can be made zero by a suitable deformation of the Hamiltonian preserving the gap. An equivalence class of a closed two-form under the addition of the differential of a globally defined one-form is called the cohomology class of this form. Thus, the cohomology class of the Berry curvature $\Omega^{(2)}$ is a topological invariant under variations of the Hamiltonian which do not close the gap.

For $D>0$, the story is similar. A topological action which does not depend on fields other than $\phi$ schematically has the form

$$
\begin{aligned}
S_{\text {top }}(X, \phi) & =\int_{X} \phi^{*}\left(\omega^{(D+1)}\right) \\
& =\frac{1}{(D+1) !} \int_{X} \omega_{i_{0} \ldots i_{D}}^{(D+1)}\left(\partial_{0} \phi^{i_{0}}\right) \ldots\left(\partial_{D} \phi^{i_{D}}\right) d x^{0} \ldots d x^{D},
\end{aligned}
$$

where $\omega^{(D+1)}$ is a $(D+1)$-form on $\mathrm{M}$ and $X$ is a closed oriented $(D+1)$-manifold. If one takes this formula literally, then all such actions can be deformed to zero, since any $(D+1)$-form can be deformed to zero. But if one interprets $\omega^{(D+1)}$ more creatively, as a sort of "higher connection," one can get more interesting actions which cannot be deformed to the trivial one. One way to find such a generalization is to note that the right-hand side (RHS) of the above equation does not change under $\omega^{(D+1)} \mapsto \omega^{(D+1)}+d \lambda^{(D)}$, where $\lambda^{(D)}$ is an arbitrary $D$-form. Then it is natural to consider an object specified by locally defined $(D+1)$-forms $\omega_{\alpha}^{(D+1)}$, where $\alpha$ labels the charts. On the overlaps of charts, these $(D+1)$ forms are related by $D$-form gauge transformations. The full story is rather complicated since, to be able to define higher holonomy along a $(D+1)$-dimensional submanifold, one needs compatibility conditions for the gauge transformations which involve $(D-1)$-forms on triple overlaps, etc.

An alternative approach (first appearing in a mathematical paper by Cheeger and Simons [6]) is to postulate the following natural property. If $X=\partial Y$ for some $(D+2)$-manifold $Y$, and if $\phi$ extends to a map $\tilde{\phi}: Y \rightarrow \mathrm{M}$, then one must have

$$
\exp \left(i S_{\text {top }}(X, \phi)\right)=\exp \left(i \int_{Y} \tilde{\phi}^{*}\left(\Omega^{(D+2)}\right)\right)
$$

where $\Omega^{(D+2)}$ is a $(D+2)$-form on M. For this formula to make sense, $\Omega^{(D+2)}$ must be closed and its periods must be integer multiples of $2 \pi$. For example, to see that $\Omega^{(D+2)}$ must be closed, one can vary $\tilde{\phi}$ infinitesimally while keeping its boundary value $\phi$ fixed. It is easy to see that the RHS will be unchanged only if $d \Omega^{(D+2)}=0$. To see that $\Omega^{(D+2)}$ must have periods which are integral multiples of $2 \pi$, take $X$ to be the empty manifold and take $Y$ to be any closed $(D+2)$ manifold.

SS one can write $\Omega^{(D+2)}=d \omega^{(D+1)}$. If the cohomology class of $\Omega^{(D+2)}$ is trivial, one can do it globally, and then $S_{\text {top }}(X, \phi)$ can be defined by the simple formula Eq. (3). In general, one can show that given a closed $(D+2)$-form $\Omega^{(D+2)}$ with quantized periods, there exists an exponentiated action $\exp \left(i S_{\text {top }}(X, \phi)\right)$ satisfying the above equation. It is unique up to a factor $\exp \left(i \int_{X} \phi^{*}(\alpha)\right)$, where $\alpha$ is a closed $(D+1)$-form on $\mathrm{M}$.

As in the case $D=0$, this implies that the cohomology class of $\Omega^{(D+2)}$ determines $\exp \left(i S_{\text {top }}(X, \phi)\right)$ up to a factor which can be deformed to 1 . Thus, one can say that deformation classes of such topological actions (known as WessZumino-Witten terms) are classified by quantized cohomology classes of degree $D+2$. There is also an interpretation of Wess-Zumino-Witten terms as holonomies of higher connections on "higher bundles" on M. Then the cohomology class of $\Omega^{(D+2)}$ determines the topology of the corresponding higher bundle. But since such an interpretation is quite abstract, we will not use it in this paper.

The conclusion is that, given a family of trivial gapped systems in spatial dimension $D$, one should be able to obtain a closed $(D+2)$-form on the parameter space with quantized periods. While the form itself depends on the dynamical details, its cohomology class is a topological invariant. It classifies possible deformation classes of Wess-Zumino-Witten terms on the parameter space.

The statement about quantization of periods needs some qualification in the case of fermionic systems. A fermionic path-integral depends on the spin structure on $X$ (i.e., choice of periodic/antiperiodic boundary conditions for fermions going around each loop). For fermionic systems, it is unreasonable to restrict attention to topological terms which depend only on the map $\phi$; one needs to study topological terms which depend both on $\phi$ and the spin structure. Then one needs to generalize the Cheeger-Simons approach by requiring the manifolds $X$ and $Y$ to be spin manifolds. Such spin-structuredependent Wess-Zumino-Witten terms were first considered in Ref. [7]. Alternatively, if one limits oneself to the case of systems on $X=\mathbb{R}^{D+1}$ or its one-point compactification $S^{D+1}$, then one can always take $Y=B^{D+2}[(D+2)$-dimensional ball]. Then the quantization condition is relaxed: Only integrals of the form

$$
\int_{S^{D+2}} h^{*}\left(\Omega^{(D+2)}\right)
$$

need to be integral multiples of $2 \pi$. Here $h: S^{D+2} \rightarrow \mathrm{M}$ is any smooth map. We will call such an $h$ a spherical cycle. Thus, for fermionic systems, only integrals of $\Omega^{(D+2)}$ over spherical cycles are quantized. Of course, not all topological terms which are consistent on $\mathbb{R}^{D+1}$ or $S^{D+1}$ will remain consistent when considered on a general space-time. That is, quantization on spherical cycles is not enough to make the Wess-Zumino-Witten action well-defined on arbitrary spin manifolds. 


\section{HIGHER BERRY CURVATURE FOR GAPPED 1D SYSTEMS}

As explained in the previous section, given a family of trivial gapped theories on a $D$-dimensional lattice and assuming that the field-theory description applies at each point in the parameter space $M$, there should be a way to construct a closed $(D+2)$-form on $\mathrm{M}$ whose integrals over spherical cycles are quantized. The cohomology class of the form is a topological invariant of the family (cannot change under deformations). In this section, we construct such a closed form $\Omega^{(3)}$ on the parameter space for the case of gapped spin chains, that is, gapped lattice $D=1$ systems. We do not use the existence of the field-theory limit. In Appendix A, we argue that integrals of $\Omega^{(3)}$ over spherical three-cycles are quantized. That is, integrals of the form $\int_{S^{3}} h^{*} \Omega^{(3)}$, where $h$ is a map from $S^{3}$ to $\mathrm{M}$, are integer multiples of $2 \pi$.

To begin, let us recall how the Berry two-form is defined for gapped 0D systems and why this definition does not work for $D>0$. Let $G=1 /(z-H)$ be the Green's function for a positive bounded Hamiltonian $H$ which depends on some parameters. Assume that 0 is an isolated eigenvalue of $H$ for all values of the parameters. Let

$$
\Omega^{(2)}=\frac{i}{2} \oint \frac{d z}{2 \pi i} \operatorname{Tr}\left(G d H G^{2} d H\right),
$$

where $\oint$ is the counterclockwise contour integral around $z=0$ and $d$ denotes the exterior derivative on the parameter space M. That is, $d=\sum_{\ell} d \lambda^{\ell} \frac{\partial}{\partial \lambda^{\ell}}$, where $\lambda^{\ell}$ are parameters. The wedge product of forms $\wedge$ is implicit in Eq. (6). $\Omega^{(2)}$ is a closed two-form on M. Indeed, since $d G=G d H G$, we compute

$$
\begin{aligned}
d \Omega^{(2)}= & \frac{i}{2} \oint \frac{d z}{2 \pi i} \operatorname{Tr}\left(G d H G d H G^{2} d H\right. \\
& \left.-G d H G^{2} d H G d H-G d H G d H G^{2} d H\right) \\
= & -\frac{i}{2} \oint \frac{d z}{2 \pi i} \operatorname{Tr}\left(G d H G^{2} d H G d H\right) \\
= & \frac{i}{6} \oint \frac{d z}{2 \pi i} \frac{\partial}{\partial z} \operatorname{Tr}(G d H G d H G d H)=0 .
\end{aligned}
$$

$\Omega^{(2)}$ is the usual Berry curvature, as one can verify by inserting a complete set of states.

Suppose now $H$ is a many-body Hamiltonian for an infinite 1D lattice system with an energy gap. More explicitly, we assume that $H=\sum_{p \in \Lambda} H_{p}$, where $H_{p}$ is bounded and finite ranged and $\Lambda \subset \mathbb{R}$ is a discrete subset of real numbers without accumulation points. Then $H$ is unbounded, but one can still define a bounded operator $G=1 /(z-H)$ for $z$, which is away from the spectrum of $H$. We assume again that $H$ is positive and that 0 is an isolated eigenvalue for all values of the parameters. Fixing $p, q \in \Lambda$, we can define a nonclosed two-form on the parameter space:

$$
\Omega_{p q}^{(2)}=\frac{i}{2} \oint \frac{d z}{2 \pi i} \operatorname{Tr}\left(G d H_{p} G^{2} d H_{q}\right) .
$$

If the Hamiltonian $H$ is gapped, $\Omega_{p q}^{(2)}$ decays exponentially away from $p=q$ (see Ref. [8]). The Berry curvature is formally given by

$$
\Omega^{(2)}=\sum_{p, q \in \Lambda} \Omega_{p q}^{(2)}
$$

but the contribution of the points near the diagonal, $p \simeq q$, is divergent for infinite-volume systems.

Instead of the ill-defined Berry curvature two-form, consider the following two-form depending on a site $p$ :

$$
F_{p}^{(2)}=\frac{i}{2} \oint \frac{d z}{2 \pi i} \operatorname{Tr}\left(G d H G^{2} d H_{p}\right) .
$$

It is well-defined but not closed. Instead one has an identity

$$
d F_{q}^{(2)}=\sum_{p \in \Lambda} F_{p q}^{(3)},
$$

where the three-form $F_{p q}^{(3)}$ is given by

$$
\begin{aligned}
F_{p q}^{(3)}= & \frac{i}{6} \oint \frac{d z}{2 \pi i} \operatorname{Tr}\left(G^{2} d H G d H_{p} G d H_{q}\right. \\
& \left.-G d H G^{2} d H_{p} G d H_{q}\right)-(p \leftrightarrow q) .
\end{aligned}
$$

The identity Eq. (9) can be verified by a straightforward computation. Note that $F_{p q}^{(3)}$ decays exponentially away from the diagonal $p=q$ thanks to the results of Ref. [8].

The identity Eq. (9) and other similar identities are key for defining topological invariants of families of gapped systems in one and higher dimensions. In the context of Euclidean lattice systems, analogous identities were first observed by Kitaev who used them to define invariants of families of such systems [2]. In this paper, we essentially derive Hamiltonian analogs of Kitaev's formulas.

Let $f: \Lambda \rightarrow \mathbb{R}$ be a function which is 0 for $p \ll 0$ and 1 for $p \gg 0$. For example, it could be simply 0 for $p<a$ and 1 for $p \geqslant a$. Then we define a three-form on the parameter space by

$$
\Omega^{(3)}(f)=\frac{1}{2} \sum_{p, q \in \Lambda} F_{p q}^{(3)}(f(q)-f(p)) .
$$

It is well-defined because on the one hand $F_{p q}^{(3)}$ decays exponentially for large $|p-q|$, and on the other hand $f(q)-f(p)$ is nonzero only when $p>a$ and $q<a$, or the other way around. For the specific choice of $f(p)$ equal 0 for $p<a$ and 1 for $p \geqslant a$, Eq. (10) takes a simple form,

$$
\Omega^{(3)}(f)=\sum_{\substack{p<a \\ q>a}} F_{p q}^{(3)},
$$

which makes its convergence more transparent.

Later in this paper, we will show that

$$
d F_{q r}^{(3)}=\sum_{p \in \Lambda} F_{p q r}^{(4)},
$$

where $F_{p q r}^{(4)}$ is a function which is antisymmetric in $p, q, r$ and decays exponentially away from the diagonal $p=q=r$. We 
find

$$
\begin{aligned}
d \Omega^{(3)}(f)= & \frac{1}{2} \sum_{q, r \in \Lambda}(f(r)-f(q)) d F_{q r}^{(3)} \\
= & \frac{1}{2} \sum_{p, q, r \in \Lambda}(f(r)-f(q)) F_{p q r}^{(4)} \\
= & \frac{1}{6} \sum_{p, q, r \in \Lambda}(f(r)-f(q)+f(p)-f(r) \\
& +f(q)-f(p)) F_{p q r}^{(4)}=0,
\end{aligned}
$$

where we have used the antisymmetry of $F_{p q r}^{(4)}$. Therefore, the three-form $\Omega^{(3)}(f)$ is closed.

Closedness of $\Omega^{(3)}(f)$ implies that its cohomology class is a topological invariant of the family of gapped systems. Indeed, let us regard $M$ as a submanifold in the space $\mathfrak{M}_{1}$ of all gapped systems in dimension 1. Obviously, the form $\Omega^{(3)}$ is a restriction of a closed form on $\mathfrak{M}_{1}$, defined in exactly the same way. Deforming $M$ within $\mathfrak{M}_{1}$ can be thought of as a flow along a vector field on $\mathfrak{M}_{1}$. Since the Lie derivative of a closed form along any vector field is exact, deforming $M$ cannot change the cohomology class of $\Omega^{(1)}$. In particular, whenever the integral over three-sphere $\int_{S^{3}} \Omega^{(3)}$ is nonzero, there must be a gapless point inside of the region of the parameter space bounded by this three-sphere.

The cohomology class of the three-form $\Omega^{(3)}(f)$ is independent of the choice of the function $f$ as long as $f(p)=0$ for $p \ll 0$ and $f(p)=1$ for $p \gg 0$. Indeed, any two such functions differ by a function $g$ which is compactly supported, and for such a function we can write

$$
\begin{aligned}
\Omega^{(3)}(g) & =\frac{1}{2} \sum_{p, q \in \Lambda}(g(q)-g(p)) F_{p q}^{(3)}=\sum_{q \in \Lambda} g(q) \sum_{p \in \Lambda} F_{p q}^{(3)} \\
& =\sum_{q \in \Lambda} g(q) d F_{q}^{(2)}=d \sum_{q \in \Lambda} g(q) F_{q}^{(2)}
\end{aligned}
$$

This means that $\Omega^{(3)}(f+g)$ and $\Omega^{(3)}(f)$ differ by a total derivative of a well-defined two-form on $\mathrm{M}$ and therefore are in the same cohomology class.

This property implies that a family of systems parameterized by a closed three-manifold $\Sigma_{3}$ with a nonzero value of the integral $\int_{\Sigma_{3}} \Omega^{(3)}(f)$ cannot have a gapped boundary condition which varies continuously over $\Sigma_{3}$. Indeed, a gapped boundary condition can be thought as an interpolation between this family for $p \gg 0$ and a trivial system for $p \ll 0$. The above result means that the integral of the three-form $\Omega^{(3)}(f)$ will be the same regardless of whether $a$ in $f(p)=\theta(p-a)$ satisfies $a \gg 0$ or $a \ll 0$. Since the formula for three-form is local, for $a \gg 0$ the integral of the three-form coincides with $\int_{\Sigma_{3}} \Omega^{(3)}(f)$ for the infinite system without the edge. On the other hand, for $a \ll 0$ it is zero. Therefore, if $\int_{\Sigma_{3}} \Omega^{(3)}(f) \neq$ 0 , the family cannot have a continuously varying boundary condition which is gapped everywhere on $\mathrm{M}$.

We note the following obvious properties of the threeform $\Omega^{(3)}(f)$ : It vanishes for constant families (i.e., families where the Hamiltonian is independent of parameters), and it is additive under stacking of families (with the same parameter space).

\section{HIGHER BERRY CURVATURE FOR GAPPED SYSTEMS IN ANY DIMENSION}

To construct analogs of Berry curvature in higher dimensions, the language of chains and cochains is very useful [9]. Let $\Lambda$ be a discrete subset of $\mathbb{R}^{D}$ without accumulation points. For $n \geqslant 0$, an $n$-chain is a quantity $A_{p_{0} \ldots p_{n}}$ which depends on $n+1$ points $p_{0}, \ldots, p_{n} \in \Lambda$, is skew symmetric under permutations of $p_{0}, \ldots, p_{n}$, and decays exponentially away from the diagonal $p_{0}=p_{1}=\ldots=p_{n}$. The space of $n$ chains will be denoted $\mathcal{C}_{n}(\Lambda)$. The boundary operator $\partial: \mathcal{C}_{n}(\Lambda) \rightarrow$ $\mathcal{C}_{n-1}(\Lambda)$ is defined as follows:

$$
(\partial A)_{p_{1} \ldots p_{n}}=\sum_{p_{0} \in \Lambda} A_{p_{0} \ldots p_{n}} .
$$

It is easy to see that $\partial^{2}=0$. Thus, $\oplus_{n \geqslant 0} \mathcal{C}_{n}(\Lambda)$ is a chain complex.

Dually, an $n$-cochain (with values in reals) is a real-valued function $\alpha\left(p_{0}, \ldots, p_{n}\right)$ which depends on $p_{0}, \ldots, p_{n} \in \Lambda$, is bounded, skew symmetric under permutations, and obeys the following condition: When restricted to any $\delta$ neighborhood of the diagonal, it vanishes when any of the points is outside some finite set. Let $\mathcal{C}^{n}(\Lambda)$ be the space of $n$ cochains. There is a pairing between $\mathcal{C}^{n}(\Lambda)$ and $\mathcal{C}_{n}(\Lambda)$ defined by

$$
\langle A, \alpha\rangle=\frac{1}{(n+1) !} \sum_{p_{0}, \ldots, p_{n}} A_{p_{0}, \ldots, p_{n}} \alpha\left(p_{0}, \ldots, p_{n}\right) .
$$

There is also an operator $\delta: \mathcal{C}^{n}(\Lambda) \rightarrow \mathcal{C}^{n+1}(\Lambda)$ satisfying $\delta^{2}=0$ and uniquely defined by the condition

$$
\langle A, \delta \alpha\rangle=\langle\partial A, \alpha\rangle
$$

for any $(n+1)$-chain $A$ and an $n$-cochain $\alpha$ and $n \geqslant 0$. One can regard Eq. (16) as a version of Stokes' theorem. Explicitly, the operator $\delta$ is given by

$$
\begin{aligned}
(\delta \alpha) & \left(p_{0}, \ldots, p_{n+1}\right) \\
& =\sum_{j=0}^{n+1}(-1)^{j} \alpha\left(p_{0}, \ldots, p_{j-1}, p_{j+1}, \ldots, p_{n+1}\right) .
\end{aligned}
$$

In particular, if $\Lambda \subset \mathbb{R}$ is a $1 \mathrm{D}$ lattice, and $f: \Lambda \rightarrow \mathbb{R}$ is a function such that $f(p)=1$ for $p \gg 0$ and $f(p)=0$ for $p \ll$ 0 , then $(\delta f)(p, q)=f(q)-f(p)$ is a closed one-cochain on $\Lambda$. It is not exact, since $f$ does not have a finite support.

One can define the product $\alpha \cup \gamma$ of an $n$-cochain $\alpha$ and an $m$-cochain $\gamma$ as an $n+m$-cochain given by

$$
\begin{aligned}
(\alpha \cup \gamma)\left(p_{0}, \ldots, p_{n+m}\right) & \\
= & \frac{1}{(n+m+1) !} \sum_{\sigma \in \mathcal{S}_{n+m+1}}(-1)^{\operatorname{sgn} \sigma} \alpha\left(p_{\sigma(0)}, \ldots, p_{\sigma(n)}\right) \\
& \times \gamma\left(p_{\sigma(n)}, \ldots, p_{\sigma(n+m)}\right) .
\end{aligned}
$$

It satisfies

$$
\begin{aligned}
\alpha \cup \gamma & =(-1)^{n m} \gamma \cup \alpha, \\
\delta(\alpha \cup \gamma) & =\delta \alpha \cup \gamma+(-1)^{n} \alpha \cup \delta \gamma .
\end{aligned}
$$

Using this notation, we see that $\Omega^{(3)}(f)=\left\langle F^{(3)}, \delta f\right\rangle$, where $F^{(3)}$ is a one-chain with values in three-forms on $\mathrm{M}$ with components $F_{p q}^{(3)}$. Furthermore, Eq. (12) can be written 
as a relation between a one-chain $F^{(3)}$ valued in three-forms and a two-chain $F^{(4)}$ valued in four-forms:

$$
d F^{(3)}=\partial F^{(4)} .
$$

Then the computation leading to Eq. (13) can be shortened to

$$
d \Omega^{(3)}(f)=\left\langle d F^{(3)}, \delta f\right\rangle=\left\langle\partial F^{(4)}, \delta f\right\rangle=\left\langle F^{(4)}, \delta \delta f\right\rangle=0 .
$$

Similarly, the computation leading to Eq. (13) can be shortened to

$$
\Omega^{(3)}(g)=\left\langle F^{(3)}, \delta g\right\rangle=\left\langle\partial F^{(3)}, g\right\rangle=d\left\langle F^{(2)}, g\right\rangle .
$$

Here $g: \Lambda \rightarrow \mathbb{R}$ is supported on a finite set, therefore the application of the Stokes' theorem is legitimate.

Now we will generalize the construction of the previous section to arbitrary dimensions and define a closed $(D+2)$ form $\Omega^{(D+2)}$ on the parameter space of a family of gapped lattice systems in $D$ spatial dimensions. We define a family of $D$-dimensional gapped lattice systems in the same way as for $D=1$, the only difference being that the lattice $\Lambda$ is a subset of $\mathbb{R}^{D}$ instead of $\mathbb{R}$. For $D>1$, not all gapped systems can be continuously connected to the trivial one, thanks to the possibility of topological order. Therefore, we do not expect our $(D+2)$-form to have quantized periods, even on spherical cycles. Nevertheless, we will argue in Appendix A that, for families of systems in an SRE phase, its periods are quantized on spherical $(D+2)$-cycles, as expected from the field-theory analysis.

We will define higher Berry curvatures recurrently via the following "descent equation":

$$
d F^{(n)}=\partial F^{(n+1)},
$$

where $F^{(n)}$ is $(n-2)$-chain with values in $n$-forms on the parameter space. Analogous equations for families of Euclidean lattice systems were used in Ref. [2]. Starting from $F^{(2)}$ defined in Eq. (9), we can find all its descendants. The result is

$$
\begin{aligned}
F_{p_{0} \ldots p_{n-2}}^{(n)}= & \frac{i(-1)^{n}}{n(n-1)} \sum_{\sigma \in \mathcal{S}_{n-1}} \operatorname{sgn}(\sigma) \oint \frac{d z}{2 \pi i} \\
& \times \sum_{j=0}^{n-2}(n-j-1) \operatorname{Tr}\left(G d H G d H_{p_{\sigma(0)}} G d H_{p_{\sigma(1)}}\right. \\
& \left.\times \ldots G^{2} d H_{p_{\sigma(j)}} \ldots G d H_{\left.p_{\sigma(n-2)}\right)}\right) .
\end{aligned}
$$

For this to be a well-defined chain, it must decay exponentially when any two of the points $p_{0}, \ldots, p_{n-2}$ are separated by a large distance. For $n=3$, this was proved in Ref. [8], and we expect that the proof can be generalized to arbitrary $n$. Heuristically, exponential decay follows from the physical interpretation of the above correlators in terms of generalized local susceptibilities. For $n=2$, the correlator is a variation of the expectation value of a local operator $d H_{p_{0}}$ with respect to an arbitrary infinitesimal variation of the Hamiltonian. That is, it is a local susceptibility. For $n=3$, it can be interpreted as a variation of a local susceptibility with respect to a variation of the Hamiltonian elsewhere. For $n=4$, it can be interpreted as a variation of a variation, etc. We expect all such quantities to decay exponentially for large spatial separations because the correlation length is finite for a gapped system at zero temperature.

To find a topological invariant of a family of gapped systems we need to contract this $(n-2)$-chain with an $(n-$ 2 )-cochain. Let $\alpha$ be an $(n-2)$-cochain, then $\left\langle F^{(n)}, \alpha\right\rangle$ is an $n$-form on the parameter space. But, in general, it is not closed:

$$
d\left\langle F^{(n)}, \alpha\right\rangle=\left\langle d F^{(n)}, \alpha\right\rangle=\left\langle\partial F^{(n+1)}, \alpha\right\rangle=\left\langle F^{(n+1)}, \delta \alpha\right\rangle .
$$

For the integral of the $n$-form $\int_{C_{n}}\left\langle F^{(n)}, \alpha\right\rangle$ to be independent of the deformation of the cycle $C_{n}$, the cochain $\alpha$ must be closed, $\delta \alpha=0$. On the other hand, if the cochain $\alpha$ is exact, $\alpha=\delta \gamma$, we find

$$
\left\langle F^{(n)}, \alpha\right\rangle=\left\langle F^{(n)}, \delta \gamma\right\rangle=\left\langle\partial F^{(n)}, \gamma\right\rangle=d\left\langle F^{(n-1)}, \gamma\right\rangle,
$$

and all integrals $\int_{C_{n}}\left\langle F^{(n)}, \alpha\right\rangle$ over cycles $C_{n}$ will be zero.

We see that to get a nontrivial invariant of a family, we need to contract the chain $F^{(n)}$ with a cochain which is closed but not exact. Moreover, adding to such a cochain, an exact cochain will not change the invariant. Thus we need to understand the space of closed cochains modulo the subspace of exact cochains, that is, the cohomology of the cochain complex $\left(\mathcal{C}^{n}(\Lambda), \delta\right)$. If we omit the word "bounded" from the definition of cochains, then the cohomology of the corresponding complex is known in the mathematical literature as the coarse cohomology of $\Lambda$ [10]. For physical applications, one may assume that $\Lambda \subset \mathbb{R}^{D}$ uniformly fills the whole $\mathbb{R}^{D}$, in the sense that there exists $\delta>0$ such that each point of $\mathbb{R}^{D}$ is within distance $\delta$ of some point of $\Lambda$, and that $\Lambda$ has no accumulation points. Then the $n$th coarse cohomology group of $\Lambda$ is isomorphic to the $n$th cohomology group of $\mathbb{R}^{D}$ with compact support [10]. The latter is nontrivial only for $n=D$ and is one-dimensional. The generator of $D$ th coarse cohomology group can be taken to be $\delta f_{1} \cup \cdots \cup \delta f_{D}$, where $f_{\mu}(p)=\theta\left(x^{\mu}(p)\right)$ and $x^{\mu}(p)$ is the $\mu$ coordinate of $p$ and $\theta(x)$ is theta function. More generally, one can choose $f_{\mu}$ to be any function which depends only on $x^{\mu}(p)$ and is 0 for $x^{\mu}(p) \ll 0$ and 1 for $x^{\mu}(p) \gg 0$. Note that such cochains are bounded and thus also define a nontrivial cohomology class in the sense that we need. For a family of $D$-dimensional systems parameterized by $\mathrm{M}$, we therefore define a $(D+2)$-form on $\mathrm{M}:$

$$
\Omega^{(D+2)}\left(f_{1}, \ldots, f_{D}\right)=\left\langle F^{(D+2)}, \delta f_{1} \cup \cdots \cup \delta f_{D}\right\rangle .
$$

This $(D+2)$-form is closed:

$$
\begin{aligned}
d \Omega^{(D+2)}\left(f_{1}, \ldots, f_{D}\right) & =\left\langle d F^{(D+2)}, \delta f_{1} \cup \cdots \cup \delta f_{D}\right\rangle \\
& =\left\langle\partial F^{(D+3)}, \delta f_{1} \cup \cdots \cup \delta f_{D}\right\rangle \\
& =\left\langle F^{(D+3)}, \delta\left(\delta f_{1} \cup \cdots \cup \delta f_{D}\right)\right\rangle=0 .
\end{aligned}
$$

Its cohomology class is unchanged under the shift $f_{1} \rightarrow f_{1}+$ $g$ by a compactly supported function $g$ since

$$
\begin{aligned}
\Omega^{(D+2)}\left(g, f_{2}, \ldots, f_{D}\right) & =\left\langle F^{(D+2)}, \delta g \cup \delta f_{2} \cup \cdots \cup \delta f_{D}\right\rangle \\
& =\left\langle F^{(D+2)}, \delta\left(g \cup \delta f_{2} \cup \cdots \cup \delta f_{D}\right)\right\rangle \\
& =\left\langle\partial F^{(D+2)}, g \cup \delta f_{2} \cup \cdots \cup \delta f_{D}\right\rangle \\
& =d\left\langle F^{(D+1)}, g \cup \delta f_{2} \cup \cdots \cup \delta f_{D}\right\rangle
\end{aligned}
$$

and analogously for other shifts $f_{\mu} \rightarrow f_{\mu}+g$. 
In general, periods of $\Omega^{(D+2)}\left(f_{1}, \ldots, f_{D}\right)$ are not subject to quantization. In Appendix A, we argue that integrals over spherical cycles in the parameter space are quantized for families of SRE systems.

\section{DISCUSSION}

In this paper, we have constructed higher-dimensional generalizations of the Berry curvature starting from the ordinary Berry curvature for quantum-mechanical systems and solving the descent Eq. (20). In fact, this procedure of constructing higher-dimensional generalization of topological invariants from lower dimensional ones via descent equations is rather general. For example, the Thouless charge pump for 1D systems [11] and its higher-dimensional generalizations can be constructed from the ground-state charge of a quantummechanical system with a $U(1)$ symmetry. This will be discussed in a separate publication.

When the usual Berry curvature of a family of gapped 0D systems is not exact, it is not possible to find continuously ground states for the whole family. Similarly, we have shown that for a family of gapped $D$-dimensional systems the cohomology class of the form $\Omega^{(D+2)}$ is an obstruction to having a continuously varying gapped boundary. Such a behavior is particularly striking when all the systems in the family are in a trivial phase. An example of a three-parameter family of 1D lattice systems in a trivial phase where $\Omega^{(3)}$ is not exact is given in Appendix $\mathrm{B}$, where we relate the three-form $\Omega^{(3)}(f)$ to the Berry-Bloch connection over the Brillouin zone.

For $D=0$, the cohomology class of the Berry curvature (regarded as an integral class) is the only topological invariant of the family. It is trivial if and only if the family can be deformed to a constant family without closing the gap. One can ask if the same is true for $D>0$ or if there are additional independent invariants. The existence of topological order for $D>1$ means that the answer will probably depend on which topological phase one considers. The case $D=1$ is special since all gapped 1D systems are SRE. Moreover, for $D=1$ it has been conjectured by Kitaev that a properly defined space of all gapped bosonic systems has the homotopy type $K(\mathbb{Z}, 3)$. That is, its only nontrivial homotopy group is in degree 3 and is isomorphic to $\mathbb{Z}$. If this is true, then all cohomology classes on the space of gapped bosonic 1D systems can be expressed as some complicated functions of the basic class which sits in degree 3. That is, for $D=1$ bosonic families there are no further independent invariants beyond the one we constructed.

\section{ACKNOWLEDGMENTS}

A.K. would like to thank D. Freed, M. Freedman, M. Hopkins, A. Kitaev. G. Moore, and C. Teleman for discussions of family invariants of gapped systems and related issues, and P.-S. Hsin and R. Thorngren for collaboration on a related project. We are especially grateful to A. Kitaev for reading a preliminary draft of the paper and pointing out an error. This research was supported in part by the US Department of Energy, Office of Science, Office of High Energy Physics, under Award No. DE-SC0011632. A.K. was also supported by the Simons Investigator Award.

\section{APPENDIX A: QUANTIZATION OF HIGHER BERRY CURVATURES}

Consider a family of gapped systems in spatial dimension $D$. In the body of the paper, we showed how to define a closed form $\Omega^{(D+2)}$ on the parameter space M. It depends on some additional data ( $D$ functions on $\Lambda$ ), but the cohomology class was shown to be independent of these data. Thus, periods of $\Omega^{(D+2)}$ are also independent of these additional data. In this Appendix, we argue that if all systems in the family are SRE, and if $h$ is a spherical cycle in M (i.e., a map $h: S^{D+2} \rightarrow \mathrm{M}$ ), then the integral of $\Omega^{(D+2)}$ over such a cycle is quantized:

$$
\frac{1}{2 \pi} \int_{S^{D+2}} h^{*}\left(\Omega^{(D+2)}\right) \in \mathbb{Z} .
$$

We begin with the $1 \mathrm{D}$ case, where there is no topological order, and thus all gapped systems without spontaneous symmetry breaking are SRE. Thus, all systems in the family belong to the same SRE phase. In the bosonic case, this means that they can all be deformed to a trivial system whose Hamiltonian is a sum of one-site operators and the ground state is a product state. In the fermionic case, there is a unique nontrivial SRE phase corresponding to Kitaev's Majorana chain. So, there are two options: Either all systems in the family are in the trivial phase or they can all be deformed to the Majorana chain. In the latter case, we can stack the whole family with the "constant" Majorana chain and get a family of fermionic systems in the trivial phase. Since $\Omega^{3}(f)$ is unchanged under stacking the family with a system independent of parameters, this reduces the problem to studying a family of systems in the trivial phase.

Let $f(p)=\theta(p)$ (a step function on $\Lambda \subset \mathbb{R})$. Recall that we denote the space of all gapped $1 \mathrm{D}$ system by $\mathfrak{M}_{1}$. (Our argument will be the same for bosonic and fermionic systems, so we do not need to distinguish the two possibilities). This is an infinite-dimensional space which can be thought of as a union of an infinite number of finite-dimensional manifolds. The parameter space $M$ is a submanifold in this infinite-dimensional space, and the three-form $\Omega^{(3)}$ on $M$ is a restriction of the three-form on $\mathfrak{M}_{1}$ defined in exactly the same way. Let us fix a particular trivial system $\mathbf{m}_{0} \in \mathfrak{M}_{1}$. Each point in $\mathrm{M}$ can be connected to $\mathbf{m}_{0}$ by a continuous path in $\mathfrak{M}_{1}$. This applies to all points in the image of the spherical cycle $h$. If this could be done continuously over the whole $S^{3}$, it would mean that the cycle is contractable to a point $\mathbf{m}_{0}$ in $\mathfrak{M}_{1}$, and the corresponding integral $\int_{S^{3}} h^{*}\left(\Omega^{(3)}(f)\right)$ would be zero. While, in general, it is not possible to contract the whole spherical cycle, it is always possible to contract $S^{3}$ with a point removed. In particular, it is possible to contract $S^{3}$ without either north or south pole. Let $S_{S}^{3}$ and $S_{N}^{3}$ be $S^{3}$ with the north and south poles removed, respectively. Let us denote the contractions in the space of the gapped Hamiltonians by $\mathcal{P}_{S}$ and $\mathcal{P}_{N}$. These are continuous maps from $[0,1] \times S_{S}^{3}$ to $\mathfrak{M}_{1}$ and from $[0,1] \times S_{N}^{3}$ to $\mathfrak{M}_{1}$, respectively. Let us parametrize $[0,1]$ by $t$. For $t=0$, they are just restrictions of $h$ to $S_{S}^{3}$ and $S_{N}^{3}$. For $t=1$, they are constant maps to $\mathbf{m}_{0}$.

Let the Hamiltonian corresponding to a point $\mathbf{m} \in \mathfrak{M}_{1}$ be $H(\mathbf{m})=\sum_{p} H_{p}(\mathbf{m})$. The family of Hamiltonians corresponding to the spherical cycle $h$ is $H[\mathbf{s}]=\sum_{p} H_{p}(h(\mathbf{s}))$, where $\mathbf{s} \in S^{3}$. For $\mathbf{s} \in S_{N}^{3}$, we define another Hamiltonian 
$H^{+}$[s] which is the same as $H[\mathbf{s}]$ except that on the far right part of the lattice $p \gg 0$ it adiabatically interpolates to $H\left(\mathbf{m}_{0}\right)$. More precisely, $H^{+}[\mathbf{s}]=\sum_{p \in \Lambda} H_{p}^{+}[\mathbf{s}]$ is sum of on-site Hamiltonians $H_{p}^{+}[\mathbf{s}]=H_{p}(\mathbf{m}(\mathbf{s}, p))$ where we let the parameters of the Hamiltonian depend slowly on $p$ as $\mathbf{m}(\mathbf{s}, p)=\mathcal{P}_{N}\left(t_{N}(p), \mathbf{s}\right)$. The function $t_{N}: \mathbb{R} \rightarrow \mathbb{R}$ is equal to 1 for $p \in[2 L,+\infty)$, smoothly interpolates from 1 to 0 in the region $p \in[L, 2 L]$, and is 0 for $p \in(-\infty, L]$. Similarly, we define a local Hamiltonian $H^{-}[\mathbf{s}]$ for all $\mathbf{s} \in S_{S}^{3}$ via $H^{-}[\mathbf{s}]=$ $\sum_{p \in \Lambda} H_{p}\left(P_{S}\left(t_{S}(p), \mathbf{s}\right)\right)$ where the function $t_{S}: \mathbb{R} \rightarrow \mathbb{R}$ is 1 for $p \in(-\infty,-2 L]$, smoothly interpolates from 1 to 0 in the region $p \in[-2 L,-L]$, and is 0 for $p \in[-L,+\infty)$. Lastly, we define $H_{p}^{+-}[\mathbf{s}]$ for all $\mathbf{s} \in S_{N}^{3} \bigcap S_{S}^{3}$ as a Hamiltonian which coincides with $H_{p}[\mathbf{s}]$ in the region $p \in[-L, L]$, coincides with $H_{p}\left(\mathbf{m}_{0}\right)$ for $p \notin[-2 L, 2 L]$, and smoothly interpolates between these regions using the paths $\mathcal{P}_{S}$ and $\mathcal{P}_{N}$. Our main assumption is that all these families of Hamiltonians are gapped for sufficiently large $L$. This seems reasonable since for a fixed $t$ and $\mathbf{s}$ all Hamiltonians $H\left(P_{N}(t, \mathbf{s})\right)$ and $H\left(P_{S}(t, \mathbf{s})\right)$ are gapped and there should be an upper bound on the correlation length. However, a proof of this would be very desirable. We denote by $\Omega_{+}^{(3)}(f), \Omega_{-}^{(3)}(f)$ and $\Omega_{+-}^{(3)}(f)$ the three-forms corresponding to the families $\mathrm{H}^{+}, \mathrm{H}^{-}$and $H^{+-}$. They are defined on $S_{N}^{3}, S_{S}^{3}$ and $S_{N}^{3} \bigcap S_{S}^{3}$, respectively.

We write an integral over $S^{3}$ as a sum of integrals over its lower and upper hemispheres, which we call $B_{-}$and $B_{+}$:

$$
\begin{aligned}
\int_{S^{3}} h^{*}\left(\Omega^{(3)}(f)\right) & =\int_{B_{+}} h^{*}\left(\Omega^{(3)}(f)\right)+\int_{B_{-}} h^{*}\left(\Omega^{(3)}(f)\right) \\
& =\int_{B_{+}} \Omega_{+}^{(3)}(f)+\int_{B_{-}} \Omega_{-}^{(3)}(f)+O\left(L^{-\infty}\right)
\end{aligned}
$$

In the last step, we replaced $h^{*}\left(\Omega^{(3)}\right)$ with $\Omega_{ \pm}^{(3)}$ on $B_{ \pm}$. Since by our assumption $H[\mathbf{s}], H^{+}[\mathbf{s}]$, and $H^{-}$[s] are all gapped, the three-form $h^{*}\left(\Omega^{(3)}\right)$ is only sensitive to the Hamiltonian of the system in the neighborhood of the point $p=0$ where the function $f(p)=\theta(p)$ has a discontinuity. Since all these Hamiltonians coincide near the point $p=0$, for large $L$ the error introduced by this replacement is of order $L^{-\infty}$.

Let us now define $f_{+}(p)=\theta(p-3 L)$ and $f_{-}(p)=\theta(p+$ $3 L$ ) and write

$$
\begin{aligned}
\int_{B_{+}} & \Omega_{+}^{(3)}(f)+\int_{B_{-}} \Omega_{-}^{(3)}(f) \\
= & \int_{B_{+}} \Omega_{+}^{(3)}\left(f_{+}\right)+\int_{B_{-}} \Omega_{-}^{(3)}\left(f_{-}\right) \\
& +\int_{B_{+}} \Omega_{+}^{(3)}\left(f-f_{+}\right)+\int_{B_{-}} \Omega_{-}^{(3)}\left(f-f_{-}\right) .
\end{aligned}
$$

The on-site Hamiltonian $H_{p}^{+}[\mathbf{s}]$ coincides with the constant Hamiltonian $H_{p}\left(\mathbf{m}_{0}\right)$ near $p=3 L$. Therefore, the form $\Omega_{+}^{(3)}\left(f_{+}\right)$is of order $L^{-\infty}$, and so is its integral over $B_{+}$. Similarly, $\int_{B_{-}} \Omega_{-}^{(3)}\left(f_{-}\right)=O\left(L^{-\infty}\right)$. The remaining terms in the above equation contain functions $f_{ \pm}-f$ which have compact support. For any such function $g: \Lambda \rightarrow \mathbb{R}$, we can write $\Omega_{ \pm}^{(3)}(g)=\left\langle F_{ \pm}^{(3)}, \delta g\right\rangle=d\left\langle F_{ \pm}^{(2)}, g\right\rangle$. Therefore, we get

$$
\begin{aligned}
\int_{B_{+}} & \Omega_{+}^{(3)}\left(f-f_{+}\right)+\int_{B_{-}} \Omega_{-}^{(3)}\left(f-f_{-}\right) \\
= & \int_{S^{2}}\left\langle F_{+}^{(2)}, f-f_{+}\right\rangle-\int_{S^{2}}\left\langle F_{-}^{(2)}, f-f_{-}\right\rangle,
\end{aligned}
$$

where $S^{2}$ is the equator of $S^{3}$ and the common boundary of $B_{-}$and $B_{+}$. The minus sign arises because the orientation on $S^{2}$ induced by $B_{-}$is opposite to the one induced by $B_{+}$. We can now replace $F_{+}^{(2)}$ and $F_{-}^{(2)}$ with $F_{+-}^{(2)}$ in both integrals, since the integrands are only sensitive to the Hamiltonian of the system in the region where $H_{p}^{+}[\mathbf{s}]=H_{p}^{+-}[\mathbf{s}]$ and $H_{p}^{-}[\mathbf{s}]=$ $H_{p}^{+-}[\mathbf{s}]$. Such a replacement introduces an error of order $L^{-\infty}$. Therefore, the above expression becomes

$$
\begin{aligned}
& \int_{S^{2}}\left\langle F_{+}^{(2)}, f-f_{+}\right\rangle-\int_{S^{2}}\left\langle F_{-}^{(2)}, f-f_{-}\right\rangle \\
& =\int_{S^{2}}\left\langle F_{+-}^{(2)}, f-f_{+}\right\rangle-\int_{S^{2}}\left\langle F_{+-}^{(2)}, f-f_{-}\right\rangle+O\left(L^{-\infty}\right) \\
& =-\int_{S^{2}}\left\langle F_{+-}^{(2)}, f_{+}-f_{-}\right\rangle+O\left(L^{-\infty}\right)
\end{aligned}
$$

By construction, $H_{p}^{+-}[\mathbf{s}]=H_{p}[\mathbf{s}]$ for $p \in[-L, L]$, while $H_{p}^{+-}[\mathbf{s}]=H\left(\mathbf{m}_{0}\right)$ for $p \notin[-2 L, 2 L]$. Since outside $[-2 L, 2 L]$ the Hamiltonian is constant, that part of the system does not contribute to $F^{(2)}$ and can be discarded. What remains is a system with a finite-dimensional Hilbert space. Since $f_{+}-f_{-}=\theta(p-3 L)-\theta(p+3 L)$ and thus is equal to -1 in the region $[-2 L, 2 L]$, we have

$$
-\left\langle F_{+-}^{(2)}, f_{+}-f_{-}\right\rangle=\sum_{p \in[-2 L, 2 L]} F_{+-p}^{(2)}+O\left(L^{-\infty}\right) .
$$

This is simply the Berry curvature of this finite-dimensional system. Therefore, its integral over $S^{2}$ is an integer multiple of $2 \pi$. We conclude that

$$
\int_{S^{3}} h^{*}\left(\Omega^{(3)}(f)\right)=2 \pi n+O\left(L^{-\infty}\right), \quad n \in \mathbb{Z} .
$$

Taking the limit $L \rightarrow \infty$ we get the desired result.

In general, we proceed by induction in $D$. For $D>1$, the restriction to SRE systems is a nontrivial constraint on the kind of families we allow. Other than that, we can proceed in the same way as for $D=1$. First, we tensor with a suitable constant SRE system to reduce to the case of a family of systems in a trivial phase. Then we remove the north and south poles from $S^{D+2}$ and define three families of gapped Hamiltonians $H^{+}[\mathbf{s}], H^{-}[\mathbf{s}]$, and $H^{+-}[\mathbf{s}]$ which are defined on $S_{N}^{D+2}, S_{S}^{D+2}$ and $S_{N}^{D+2} \bigcap S_{S}^{D+2}$, respectively. They approach $H\left(\mathbf{m}_{0}\right)$ on the far right, far left, and both far right and far left, respectively. By far right, we mean the region $x^{D}(p) \gg 0$, while far left is the region $x^{D}(p) \ll 0$. The same manipulations as before reduce the integral of $\Omega^{(D+2)}$ over $S^{D+2}$ to an integral of $\Omega^{(D+1)}$ over the equatorial $S^{D+1}$ up to terms of order $L^{-\infty}$. This completes the inductive step.

An interpolation between $H(\mathbf{m})$ and $H\left(\mathbf{m}_{0}\right)$ can also be viewed as a gapped boundary condition for $H(\mathbf{m})$. Given a smooth family of gapped boundary conditions for $H$ [s] defined on some open subset $U \subset S^{3}$ (not necessarily arising from a smooth interpolation as above), one can write 
$\left.\Omega^{(D+2)}\left(f_{1}, \ldots, f_{D}\right)\right|_{U}$ as an exact form. This is done in exactly the same way as above. Therefore if the cohomology class of $\Omega^{(D+2)}$ is nontrivial, it is impossible to find a family of gapped boundary conditions for $H[\mathbf{s}]$ which is defined on the whole $S^{3}$ and varies smoothly with $\mathbf{s}$. For $D=0$, the analogous statement is that the cohomology class of the Berry curvature is an obstruction to finding a family of ground states on the whole parameter space which depends continuously on the parameters.

\section{APPENDIX B: HIGHER BERRY CURVATURE FOR 1D INSULATORS OF CLASS A}

In this Appendix, we compute the higher Berry curvature three-form in the case of gapped systems of free fermions in $1 \mathrm{D}$ with conserved charge (that is, insulators of class A). Then we specialize to the case of translationally invariant systems and compare with forms constructed out of the Bloch-Berry connection.

We start with the many-body expression for the three-form $F_{p q}^{(3)}$ divided by $2 \pi$ :

$$
\begin{aligned}
\frac{F_{p q}^{(3)}}{2 \pi}= & -\frac{i}{12 \pi} \oint_{z=E_{0}} \frac{d z}{2 \pi i} \operatorname{Tr}\left(2 G d H G^{2} d H_{p} G d H_{q}\right. \\
& \left.+G d H G d H_{p} G^{2} d H_{q}\right)-(p \leftrightarrow q) .
\end{aligned}
$$

We will consider the following many-body Hamiltonian:

$$
H_{p}=\frac{1}{2} \sum_{m \in \Lambda}\left(a_{p}^{\dagger} h(p, m) a_{m}+a_{m}^{\dagger} h(m, p) a_{p}\right) .
$$

Here $h(p, q)$ is an Hermitian matrix $h(p, q)^{*}=h(q, p)$. The fermionic creation-annihilation operators $a_{p}^{\dagger}, a_{p}$ satisfy canonical anticommutation relations,

$$
\begin{aligned}
& \left\{a_{p}^{\dagger}, a_{q}\right\}=\delta_{p q}, \\
& \left\{a_{p}, a_{q}\right\}=\left\{a_{p}^{\dagger}, a_{q}^{\dagger}\right\}=0,
\end{aligned}
$$

where $\delta_{p q}$ is the Kronecker delta.

Since all relevant operators are sums of single particle operators, matrix elements $\langle m|A| n\rangle$ vanish unless many-body states $n$ and $m$ differ by exactly one single-particle excitation. The above expression can be written in terms of one-particle quantities as follows:

$$
\begin{aligned}
\frac{F_{p q}^{(3)}}{2 \pi}= & -\frac{i}{12 \pi} \oint \frac{d z}{2 \pi i} \operatorname{tr}\left(2 g d h g^{2} d h_{p} g d h_{q}\right. \\
& \left.+g d h g d h_{p} g^{2} d h_{q}\right)-(p \leftrightarrow q) .
\end{aligned}
$$

Here the contour of integration encloses all states below Fermi level and all lowercase letters denote the corresponding single-particle operators acting on the single-particle Hilbert space $\ell^{2}(\Lambda)$. Naively, this integral contains additional contributions compared to Eq. (B1) where a fermion jumps from an empty state or jumps into a filled state. But these contributions cancel each other and the result coincides with Eq. (B1).

Hamiltonian density at a point $p$ can be written as $h_{p}=$ $\frac{1}{2}\left(\delta_{p} h+h \delta_{p}\right)$, where $\delta_{p}$ is Kronecker's delta (equal to 1 on $p$ and 0 on other sites) and functions are understood as operators on the one-particle Hilbert space acting by multiplication.
Contracting $F_{p q}^{(3)}$ with the cochain $f(q)-f(p)$, we find

$$
\begin{aligned}
\frac{1}{2 \pi} & \left\langle F^{(3)}, \delta f\right\rangle \\
= & -\frac{i}{24 \pi} \oint \frac{d z}{2 \pi i} \operatorname{tr}\left([d h, f]\left(g d h g d h g^{2}-g^{2} d h g d h g\right)\right. \\
& \left.-2[h, f]\left(g d h g^{2} d h g d h g-g d h g d h g^{2} d h g\right)\right) .
\end{aligned}
$$

Note that multiplication by $f$ is not a trace class operator, since it acts on infinitely many sites. Therefore, traces containing them are not guaranteed to exist. On the other hand, commutators like $[d h, f]$ are supported only on a finite number of sites and traces containing them are well-defined.

On the other hand, given a gapped 1D system of free fermions with translational symmetry which depends on three parameters $\lambda_{1}, \lambda_{2}, \lambda_{3}$, one may consider the Bloch bundle of filled states over the product of the Brillouin zone $S^{1}$ and the parameter space $\Sigma$. It carries the non-Abelian Bloch-Berry connection, and one can consider various Chern-Weil forms on $S^{1} \times \Sigma$ constructed out of this connection. In particular, one can consider the degree- 4 component of the Chern character of the Berry-Bloch connection and its integral over $S^{1} \times \Sigma$ :

$$
\int_{S^{1} \times \Sigma} \operatorname{Ch}(\mathcal{F})=-\frac{1}{8 \pi^{2}} \int_{S^{1} \times \Sigma} \operatorname{Tr}(\mathcal{F} \wedge \mathcal{F}) .
$$

Here $\mathcal{F}$ is the non-Abelian curvature two-form of the BlochBerry connection and trace is taken over filled bands. It can be shown (see Sec. III A in Ref. [12]) that this quantity can be expressed in terms of the one-particle Green's function as follows:

$$
\begin{aligned}
- & \frac{1}{8 \pi^{2}} \int_{S^{1} \times \Sigma} \operatorname{Tr}(\mathcal{F} \wedge \mathcal{F}) \\
= & \frac{\pi^{2}}{15} \epsilon^{\mu \nu \rho \sigma \tau} \oint \frac{d z}{2 \pi i} \int_{S_{1}} \frac{d k}{2 \pi} \int_{\Sigma} \frac{d^{3} \lambda}{(2 \pi)^{3}} \operatorname{tr}^{\prime}\left[\left(g \frac{\partial g^{-1}}{\partial q^{\mu}}\right)\right. \\
& \left.\times\left(g \frac{\partial g^{-1}}{\partial q^{\nu}}\right)\left(g \frac{\partial g^{-1}}{\partial q^{\rho}}\right)\left(g \frac{\partial g^{-1}}{\partial q^{\sigma}}\right)\left(g \frac{\partial g^{-1}}{\partial q^{\tau}}\right)\right],
\end{aligned}
$$

where $q^{\mu}=\left(z, k, \lambda_{1}, \lambda_{2}, \lambda_{3}\right)$. The first integral encloses filled levels, the second integral is over the Brillouin zone, and the last integral is over the parameter space $\Sigma$. The trace $\operatorname{tr}^{\prime}$ is taken over subspace with fixed momentum $k$. In translationally invariant systems, we can interpret $\int_{S_{1}} \frac{d k}{2 \pi}$ as part of the trace tr over the whole one-particle Hilbert space and substitute $\frac{\partial g^{-1}}{\partial k}=-\frac{\partial h}{\partial k}=-i[h, f]$. Expanding the derivatives $\partial / \partial q^{\mu}$ and combining parameter derivatives into forms, $\sum_{i} \frac{\partial h}{\partial \lambda^{i}} d \lambda^{i}=d h$, we find

$$
\begin{aligned}
- & \frac{1}{8 \pi^{2}} \int_{S^{1} \times \Sigma} \operatorname{Tr}(\mathcal{F} \wedge \mathcal{F}) \\
= & \frac{i}{24 \pi} \oint \frac{d z}{2 \pi i} \int_{\Sigma} \operatorname{tr}\left(g^{2}[h, f] g d h g d h g d h\right. \\
& -g^{2} d h g[h, f] g d h g d h+g^{2} d h g d h g[h, f] g d h \\
& \left.\quad-g^{2} d h g d h g d h g[h, f]\right) .
\end{aligned}
$$


One can see that the integrand of this expression differs from Eq. (B5) by a total derivative proportional to

$$
d\left(\oint \frac{d z}{2 \pi i} \operatorname{tr}\left([h, f]\left(g d h g d h g^{2}-g^{2} d h g d h g\right)\right)\right) .
$$

Since $\Sigma$ was an arbitrary three-dimensional submanifold of the parameter space, we have shown that the first higher Berry three-form divided by $2 \pi$ is in the same cohomology class as $\int_{S^{1} \times \Sigma} \mathrm{Ch}(\mathcal{F})$. We conjecture that, more generally for class A insulators in $D$ dimensions, the form $\Omega^{(D+2)}$ is in the same cohomology class as the integral of the degree $2 D+2$ component of the Chern character of the Bloch-Berry connection over the Brillouin zone.

An example of a free 1D fermion system with a nontrivial integral $\int_{S^{1} \times \Sigma} \mathrm{Ch}(\mathcal{F})$ can be constructed using the 4D Chern insulator (see Sec. III B of Ref. [12]). The Hamiltonian is

$$
H=\sum_{k_{x}} \psi_{k_{x}}^{\dagger} d_{a}\left(k_{x}, \vec{\lambda}\right) \Gamma^{a} \psi_{k_{x}}
$$

where $\Gamma^{a}$ are five Dirac matrices generating a Clifford algebra, and

$$
\begin{aligned}
d_{a}\left(k_{x}, \vec{\lambda}\right)= & {\left[\left(m+c+\cos k_{x}\right.\right.} \\
& \left.\left.+c \sum_{i=1}^{3} \cos \lambda_{i}\right), \sin k_{x}, \sin \lambda_{1}, \sin \lambda_{2}, \sin \lambda_{3}\right] .
\end{aligned}
$$

It was shown in Ref. [12] that if we chose $\Sigma$ to be threetorus $S^{1} \times S^{1} \times S^{1}$ defined by identification $\lambda_{i} \sim \lambda_{i}+2 \pi$, this model has a nonzero integer value of the integral $\int_{S^{1} \times S^{1} \times S^{1} \times S^{1}} \mathrm{Ch}(\mathcal{F})$ for a particular choice of $m$ and $c$. One can think about this family of $1 \mathrm{D}$ models as a dimensional reduction of the 4D Chern insulator, where we treat three out of four components of momentum as parameters.

Note that the Atiyah-Singer index theorem [13] implies that the integral of the Chern character of a vector bundle over a four-torus is an integer. Therefore the integral of $\Omega^{(3)}$ over the parameter space $T^{3}$ is $2 \pi$ times an integer, despite the fact that the parameter space is a torus rather than a sphere.
[1] M. V. Berry, Quantal phase factors accompanying adiabatic changes, Proc. R. Soc. London A 392, 45 (1984).

[2] A. Kitaev, Differential forms on the space of statistical mechanical lattice models, talk at Between Topology and Quantum Field Theory: A Conference in Celebration of Dan Freed's 60th Birthday, https://web.ma.utexas.edu/topqft/ talkslides/kitaev.pdf.

[3] V. I. Arnold, Catastrophe Theory, 2nd ed. (Springer-Verlag, Berlin, Heidelberg, 1986).

[4] C. Cordova, D. S. Freed, H. T. Lam, and N. Seiberg, Anomalies in the space of coupling constants and their dynamical applications I, SciPost Phys. 8, 001 (2020).

[5] C. Cordova, D. S. Freed, H. T. Lam, and N. Seiberg, Anomalies in the space of coupling constants and their dynamical applications II, SciPost Phys. 8, 002 (2020).

[6] J. Cheeger and J. Simons, Differential characters and geometric invariants, Geometry and Topology, Lecture Notes in Mathematics Vol. 1167 (Springer, Berlin, Heidelberg, 1985).
[7] D. S. Freed, Pions and Generalized Cohomology, J. Diff. Geom. 80, 45 (2008).

[8] H. Watanabe, Insensitivity of bulk properties to the twisted boundary condition, Phys. Rev. B 98, 155137 (2018).

[9] A. Kapustin and L. Spodyneiko, Thermal Hall conductance and a relative topological invariant of gapped two-dimensional systems, Phys. Rev. B 101, 045137 (2020).

[10] J. Roe, Lectures on Coarse Geometry (American Mathematical Society, Providence, 2003).

[11] D. J. Thouless, Quantization of particle transport, Phys. Rev. B 27, 6083 (1983).

[12] X.-L. Qi, T. L. Hughes, and S.-C. Zhang, Topological field theory of time-reversal invariant insulators, Phys. Rev. B 78, 195424 (2008).

[13] M. F. Atiyah and I. M. Singer, The index of elliptic operators on compact manifolds, Bull. Am. Math. Soc. 69, 422 (1969). 